\title{
Quantum anti-Zeno effect without rotating wave approximation
}

\author{
Qing Ai (艾清), ${ }^{1}$ Yong Li (李勇), ${ }^{2}$ Hang Zheng (郑杭), ${ }^{3}$ and C. P. Sun (孙昌璞) ${ }^{1}$ \\ ${ }^{1}$ Institute of Theoretical Physics, Chinese Academy of Sciences, Beijing 100190, People's Republic of China \\ ${ }^{2}$ Department of Physics and Center of Theoretical and Computational Physics, The University of Hong Kong, \\ Pokfulam Road, Hong Kong, People's Republic of China \\ ${ }^{3}$ Department of Physics, Shanghai Jiao Tong University, Shanghai 200030, People's Republic of China
}

(Received 9 March 2010; published 30 April 2010)

\begin{abstract}
In this article, we systematically study the spontaneous decay phenomenon of a two-level system under the influences of both its environment and repetitive measurements. In order to clarify some well-established conclusions about the quantum Zeno effect (QZE) and the quantum anti-Zeno effect (QAZE), we do not use the rotating wave approximation (RWA) in obtaining an effective Hamiltonian. We examine various spectral distributions by making use of our present approach in comparison with other approaches. It is found that with respect to a bare excited state even without the RWA, the QAZE can still happen for some cases, for example, the interacting spectra of hydrogen. However, for a physical excited state, which is a renormalized dressed state of the atomic state, the QAZE disappears and only the QZE remains. These discoveries inevitably show a transition from the QZE to the QAZE as the measurement interval changes.
\end{abstract}

DOI: 10.1103/PhysRevA.81.042116

PACS number(s): 03.65.Xp, 03.65.Yz, 42.50.Ct

\section{INTRODUCTION}

The quantum Zeno effect (QZE) is vividly described as the adage "a watched pot never boils" in some quantum version [1]. Usually it is used for describing a class of effects in which constant monitoring of a quantum system drastically slows down its dynamic evolution [1,2]. This may be a coherent transition (e.g., the Rabi oscillation [3]) and an irreversible process as well. For instance, any unstable state can be prevented from decay when adequate measurements are frequently applied to the system [4-7]. Here the couplings to a reservoir would induce an exponential decay if there were no measurements.

On the other hand, it was predicted that the decay could also be enhanced by frequent measurements observed under somewhat different conditions, leading to the so-called quantum anti-Zeno effect (QAZE) [8,9]. When the coupling to a surrounding environment (a reservoir) is taken into consideration, the generic QZE may not be attainable since the required measurement interval is out of reach in experiments. Furthermore, under the influence of the reservoir with some spectral distribution, the decay process could be significantly accelerated by repetitive measurements.

Recently it was recognized [10,11] that the theoretical prediction [8] for the reservoir-enhanced decay phenomena may be based on the rotating wave approximation (RWA) [3], where the counter-rotating terms are neglected as they are high-frequency oscillating. A quite natural question follows as to whether the existence of the QAZE really relies on the counter-rotating term, which is usually ignored in many applications since it possesses high-frequency oscillation in the interaction picture.

In this article, we generally tackle this problem by investigating the influence of the counter-rotating term on the QAZE. Without making the RWA, as done in Ref. [10], we develop a direct canonical transformation approach [12,13] to derive an effective Hamiltonian. It is equivalent to the second-order perturbation approach. The obtained effective Hamiltonian is exactly solvable since it possesses the same form as that for the case with the RWA. Our calculation properly shows that for the spontaneous decay there exists a transition from the QZE to the QAZE as the measurement interval changes. In other words, with respect to the bare excited state (the product state of the atomic excited state and the vacuum of the reservoir) in the spontaneous decay, the counter-rotating terms are irrelevant to the occurrence of the QAZE for some spectral structures. As predicted, the essential difference between these approaches with and without the RWA could be disregarded in some cases.

In addition to the spectra of the hydrogen atom, we extend our research to the general situations with different kinds of spectral structures. Our finding shows that the QAZE seems to be universal except when some certain requirement is met for a sub-Ohmic spectrum. Furthermore, in order to compare with the existing research [10], we also start from the same unitary transformation, but choose the bare excited state, which is different from the physical excited state (the one excited from the ground state of the original Hamiltonian) in Ref. [10], as the initial state. Then the QAZE is again witnessed for the cases of hydrogen's spectral structure and others as well. The discrepancy between our result and the former one [10] is attributed to the different choices of the initial states.

The article is structured as follows. In the next section, with a special transformation, we obtain the effective Hamiltonian and thus the modification of the atomic spontaneous decay rate due to the counter-rotating terms. Section III discusses the transition from the QAZE to the QZE for different spectra. In Sec. IV, with the same initial state, we start from anther transformation and arrive at the same conclusion for the hydrogen atom as the one in the previous section. A brief summary is concluded in Sec. V. Furthermore, we prove in Appendix A that the survival probability of the atom in the excited state is equivalent to the survival probability of the initial state for the spontaneous decay. In addition to Sec. IV, Appendix B presents the details on the calculation of the survival probability. 


\section{EFFECTIVE HAMILTONIAN WITHOUT ROTATING WAVE APPROXIMATION}

We generally consider the QAZE for a two-level atom interacting with a reservoir in vacuum in the weak coupling limit. According to A. O. Caldeira and A. J. Leggett [14], the reservoir weakly coupled to an open quantum system can universally be modeled as a collection of many harmonic oscillators with annihilation (creation) operator $b_{k}\left(b_{k}^{\dagger}\right)$ for $k$ th mode with frequency $\omega_{k}$. Let $\sigma_{x, y, z}$ be the Pauli operators and

$$
\sigma^{ \pm}=\frac{1}{2}\left(\sigma_{x} \pm i \sigma_{y}\right)
$$

the raising and lowering operators for the atom with the excited state $|e\rangle$, the ground state $|g\rangle$, and the energy-level spacing $\Omega$, respectively. Then the total system is described by the Hamiltonian $H=H_{0}+H_{I}$ :

$$
\begin{gathered}
H_{0}=\sum_{k} \omega_{k} b_{k}^{\dagger} b_{k}+\frac{\Omega}{2} \sigma_{z}, \\
H_{I}=\sum_{k} g_{k}\left[\left(b_{k}+b_{k}^{\dagger}\right) \sigma^{+}+\text {H.c. }\right] .
\end{gathered}
$$

Here we have assumed the coupling constants $g_{k}$ 's to be real for simplicity. However, we would like to say that the main result does not change if we start from a general assumption that $g_{k}$ 's are complex numbers.

As the interaction term $H_{I}$ contains the counter-rotating terms, that is, the high-frequency terms with frequencies $\pm\left(\omega_{k}+\Omega\right)$ like

$$
V=b_{k}^{\dagger} \sigma^{+} e^{i\left(\omega_{k}+\Omega\right) t}+\text { H.c. }
$$

in the interaction picture, the Hamiltonian $H$ is not exactly solvable even for the simple cases of single mode or single excitation. We use the generalized version [12] of the FröhlichNakajima transformation $\exp (-S)[15,16]$ to eliminate the high-frequency terms in the effective Hamiltonian. Here

$$
S=\sum_{k} A_{k}\left(b_{k}^{\dagger} \sigma^{+}-b_{k} \sigma^{-}\right)
$$

is an anti-Hermitian operator, where $A_{k}$ 's remain to be determined. Up to the second order, the effective Hamiltonian $H_{\text {eff }}=\exp (-S) H \exp (S)$ is given as

$$
H_{\text {eff }}=H_{0}+H_{1}+\frac{1}{2}\left[H_{1}, S\right]+\frac{1}{2}\left[H_{I}, S\right]+\cdots .
$$

Now we require $b_{k}^{\dagger} \sigma^{+}+$H.c. to be eliminated from the firstorder term

$$
\begin{aligned}
H_{1} & =H_{I}+\left[H_{0}, S\right] \\
& =\sum_{k}\left[g_{k}\left(b_{k}+b_{k}^{\dagger}\right) \sigma^{+}+A_{k}\left(\omega_{k}+\Omega\right) b_{k}^{\dagger} \sigma^{+}+\text {H.c. }\right] \\
& =\sum_{k} g_{k}\left(b_{k} \sigma^{+}+\text {H.c. }\right) .
\end{aligned}
$$

The preceding equation gives the coefficients

$$
A_{k}=-\frac{g_{k}}{\omega_{k}+\Omega}
$$

Note that for a state $|\Psi\rangle$ which fulfills the Schrödinger equation before the transformation, that is,

$$
H|\Psi\rangle=i \partial_{t}|\Psi\rangle,
$$

we can prove that the state after the transformation $|\Psi\rangle^{S}=$ $\exp (-S)|\Psi\rangle$ satisfies the Schrödinger equation,

$$
H_{\mathrm{eff}}|\Psi\rangle^{S}=i \partial_{t}|\Psi\rangle^{S}
$$

with the effective Hamiltonian

$$
H_{\mathrm{eff}}=\sum_{k} \omega_{k} b_{k}^{\dagger} b_{k}+\frac{\Omega_{1}}{2} \sigma_{z}+\sum_{k} g_{k}\left(b_{k} \sigma^{+}+\text {H.c. }\right),
$$

where we have omitted the high-frequency intercrossing terms such as $b_{k}^{\dagger} b_{k^{\prime}}^{\dagger}$ and $b_{k^{\prime}} b_{k}$, and the modified level spacing for the atom is

$$
\Omega_{1}=\Omega+\sum_{k} \frac{g_{k}^{2}}{\omega_{k}+\Omega}
$$

Here the shift of the level spacing can be regarded as the Lamb shift, also called the ac stark modification in atomic physics and quantum optics. Furthermore, in the preceding calculation, the term

$$
\sum_{k} \frac{g_{k}^{2}}{\omega_{k}+\Omega}\left(1+b_{k}^{\dagger} b_{k}\right)
$$

is replaced with

$$
\sum_{k} \frac{g_{k}^{2}}{\omega_{k}+\Omega}
$$

since for the single excitation case its contribution results in a small modification in the $l$ th mode $g_{l}^{2} /\left(\omega_{l}+\Omega\right)$. We remark that, for those modes $k \neq k^{\prime}$ with smaller frequency differences, the terms $b_{k}^{\dagger} b_{k^{\prime}}\left(k \neq k^{\prime}\right)$ could have larger contributions in quantum dynamics, but for some initial states we choose them to be of the second order. This problem has been considered in Ref. [10].

Let us first consider the QAZE for the spontaneous decay process where the initial state can be chosen as $|e,\{0\}\rangle=|e\rangle \otimes$ $|\{0\}\rangle$ with the atom in the excited state $|e\rangle$ and all modes of fields in the vacuum state $|\{0\}\rangle=\prod_{k} \otimes\left|0_{k}\right\rangle$. Due to the special unitary transformation $\exp (-S)$, the initial states before and after the transformation are identical; that is,

$$
e^{-S}|e,\{0\}\rangle=\left(I-S+\frac{1}{2} S^{2}\right)|e,\{0\}\rangle=|e,\{0\}\rangle .
$$

We note that, for the generalized Fröhlich-Nakajima transformation in Ref. [10], the initial state would be changed. For other cases, we illustrate that the uses of changed and unchanged initial states would result in the different conclusions about the discussions of the QAZE.

When the atom is projected onto the excited state, provided that the total system evolves from the initial state $|e,\{0\}\rangle$, the survival probability is

$$
P(t)=|x(t)|^{2}=\operatorname{Tr}\left(|e\rangle\left\langle e\left|e^{-i H t}\right| e,\{0\}\right\rangle\langle e,\{0\}| e^{i H t}\right) .
$$


Thus, as shown in Appendix A, the survival probability after $n$ measurements,

$$
\begin{aligned}
P(t=n \tau) & =\left|\left\langle e,\{0\}\left|e^{-i H \tau}\right| e,\{0\}\right\rangle\right|^{2 n} \\
& =\left|\left\langle e,\{0\}\left|e^{S} e^{-i H_{\mathrm{eff}} \tau} e^{-S}\right| e,\{0\}\right\rangle\right|^{2 n} \\
& =\left|\left\langle e,\{0\}\left|e^{-i H_{\mathrm{eff}} \tau}\right| e,\{0\}\right\rangle\right|^{2 n} \\
& =e^{-R t},
\end{aligned}
$$

is calculated in the transformed representation where the new initial state just coincides with the original one. Here the decay rate [8]

$$
R=2 \pi \int_{-\infty}^{\infty} d \omega F\left(\omega, \Omega_{1}\right) G(\omega)
$$

is the overlap integral of the measurement-induced atomic level broadening

$$
\begin{aligned}
F\left(\omega, \Omega_{1}\right) & =\frac{1}{2 \pi} \int_{-\infty}^{\infty} d t\left(1-\frac{|t|}{\tau}\right) e^{i \Omega_{1} t} \theta(\tau-|t|) e^{-i \omega t} \\
& =\frac{\tau}{2 \pi} \operatorname{sinc}^{2}\left[\frac{\left(\omega-\Omega_{1}\right) \tau}{2}\right]
\end{aligned}
$$

and the interacting spectrum

$$
G(\omega)=\int_{-\infty}^{\infty} d t \sum_{k} \frac{g_{k}^{2}}{2 \pi} e^{i\left(\omega-\omega_{k}\right) t}=\sum_{k} g_{k}^{2} \delta\left(\omega-\omega_{k}\right) .
$$

The preceding obtained result seems to be the same as that in Ref. [8], but the essential difference is that the peak of $F\left(\omega, \Omega_{1}\right)$ has been shifted due to the counter-rotating terms. In this approach for practical problems, this shift may not have significant effect on the physical result (see the illustration in Fig. 1). In the physical systems that we are considering, that is, hydrogen atom, the influence of the counter-rotating terms is very small since the energy shift $\left|\Omega_{1}-\Omega\right|$ is relatively small with respect to the distance between the original energy level spacing $\Omega$ and the peak of the interacting spectrum $\omega_{0}$. However, there may appear some different results in artificial systems such as circuit QED [17]. We check this observation for various cases as follows.

\section{QUANTUM ANTI-ZENO EFFECT FOR DIFFERENT INTERACTING SPECTRA}

Having obtained the effective decay rate modified by the counter-rotating terms, we examine the preceding observation for specific spectra in investigating the QZE and the QAZE.

\section{A. Quantum anti-Zeno effect for hydrogen atom}

Let us first investigate the decay rate for the hydrogen atom in the vacuum of electromagnetic fields. We consider two usual transitions of the hydrogen atom, that is, $2 p-1 s$ and $3 p-1 s$, with the interacting spectra $[18,19]$

$$
G_{2 p-1 s}(\omega)=\frac{\eta \omega}{\left[1+\left(\frac{\omega}{\omega_{c}}\right)^{2}\right]^{4}}
$$

and

$$
G_{3 p-1 s}(\omega)=\frac{\eta^{\prime} \omega\left[1+2\left(\frac{\omega}{\omega_{c}^{\prime}}\right)^{2}\right]^{2}}{\left[1+\left(\frac{\omega}{\omega_{c}^{\prime}}\right)^{2}\right]^{6}}
$$

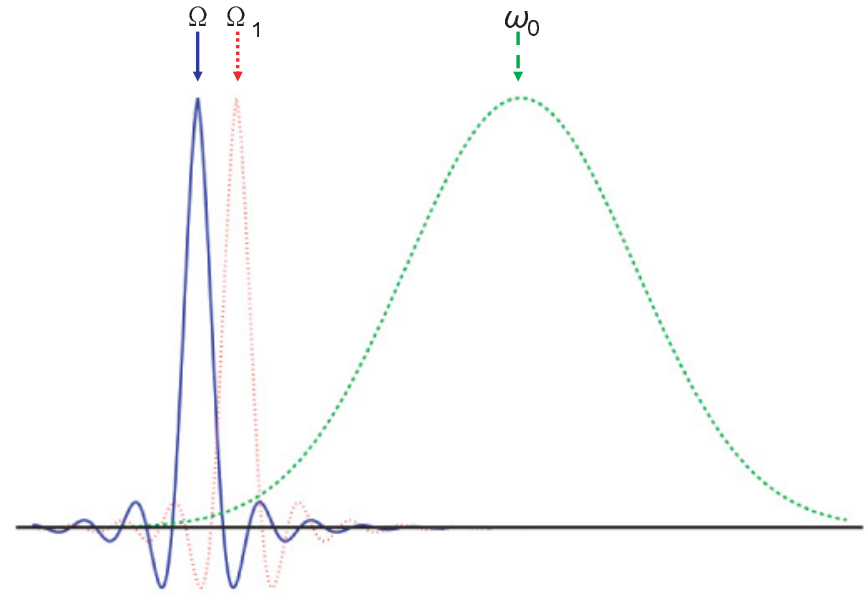

FIG. 1. (Color online) Schematic of the difference between the overlap integrals with and without the RWA. The solid blue line is for the measurement function $F(\omega, \Omega)$ centered at the original atomic level spacing $\Omega$ for the case with the RWA, the dotted red line is for the measurement function $F\left(\omega, \Omega_{1}\right)$ centered at the modified frequency $\Omega_{1}$ for our current case without the RWA, and the green dashed line is for interacting spectrum $G(\omega)$ centered at $\omega_{0}$.

respectively, where

$$
\begin{gathered}
\eta=6.435 \times 10^{-9}, \quad \omega_{c}=8.491 \times 10^{18} \mathrm{rad} / \mathrm{s} \\
\eta^{\prime}=1.455 \times 10^{-9}, \quad \omega_{c}^{\prime}=7.547 \times 10^{18} \mathrm{rad} / \mathrm{s} .
\end{gathered}
$$

The numerical calculations of the decay rate in Eq. (13) are shown in Figs. 2 and 3. Here we observe the emergence of the QZE and the QAZE as well. Starting from a large-enough value of $\tau$, as the measurement interval decreases, the decay rate will experience an ascending procedure at the first stage. Since the decay rate is bigger than the unperturbed one

$$
R_{0}=2 \pi G\left(\Omega_{1}\right),
$$

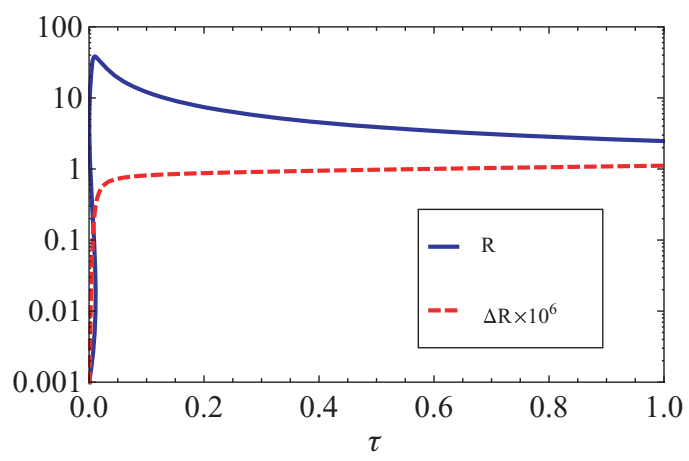

FIG. 2. (Color online) The decay rate vs measurement interval $\tau$ for the $2 p-1 s$ transition of the hydrogen atom. Here the solid blue line is for $R$ and the dashed red line is for $\Delta R=\left|R-R_{\mathrm{rwa}}\right|$. $\left|\Omega_{1}-\Omega\right| / \Omega=1.71 \times 10^{-6}, \Omega=1.55 \times 10^{16} \mathrm{rad} / \mathrm{s}$, and $\omega_{c} / \Omega=$ 550. Notice that $\Delta R$ is enlarged $10^{6}$ times. In all figures, the measurement interval $\tau$ is in units of atomic level spacing $1 / \Omega$ and the decay rate $R$ is normalized with respect to the unperturbed one $R_{0}$. 


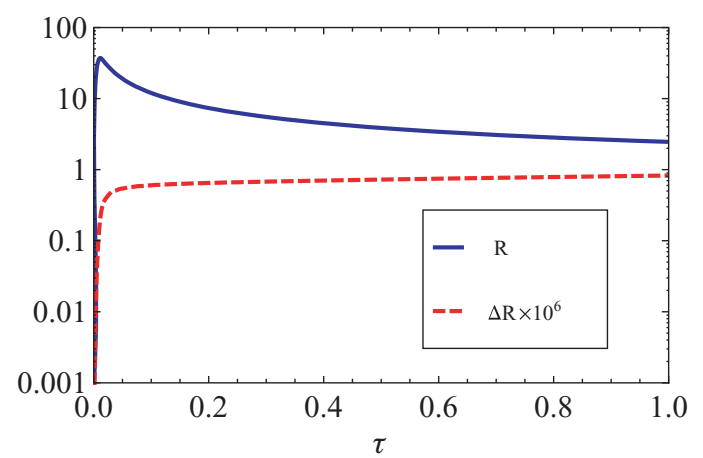

FIG. 3. (Color online) The decay rate vs measurement interval $\tau$ for the $3 p-1 s$ transition of the hydrogen atom. Here the solid blue line is for $R$ and the dashed red line is for $\Delta R=\left|R-R_{\mathrm{rwa}}\right|$. $\left|\Omega_{1}-\Omega\right| / \Omega=1.27 \times 10^{-6}, \Omega=1.83 \times 10^{16} \mathrm{rad} / \mathrm{s}$, and $\omega_{c}^{\prime} / \Omega=$ 412. Notice that $\Delta R$ is enlarged by $10^{6}$ times.

the QAZE occurs before it reaches the climax. After the turning point, the trend is changed. It is obvious that the decay rate drops steeply as the $\tau$ is further reduced. When the normalized decay rate falls below 1, the QZE is present. As the measurement becomes more and more frequent, that is, $\tau \rightarrow 0$, we observe the transition from the QAZE to the QZE. Mathematically speaking, the decay rate is the overlap integral of the measurement-induced atomic level broadening $F(\omega, \Omega)$ and the interacting spectrum $G(\omega) . F(\omega, \Omega)$ is peaked at $\Omega$ with width $1 / \tau$ while $G(\omega)$ is maximized at a frequency of the order of the cutoff frequency $\omega_{c}$ which is much bigger than the atomic level spacing $\Omega$. As $\tau$ decreases from a large-enough value, $F(\omega, \Omega)$ covers more and more the increasing part of $G(\omega)$. As a consequence, the decay rate is enhanced and the QAZE is witnessed. When the measurement interval $\tau$ is reduced to the order of $1 / \omega_{c}$, the decay rate will no longer increase since $F(\omega, \Omega)$ has already covered the main part of $G(\omega)$. Afterward, the opposite phenomenon is observed. In these two figures, also shown are the differences between the decay rates obtained from the one with the RWA and the one without the RWA, $\Delta R=\left|R-R_{\text {rwa }}\right|$. Notice that $\Delta R$ 's are of the order of $10^{-6}$ (in units of $R_{0}$ ). It is a reasonable result since the only effect of the counter-rotating terms lies in the modified level spacing $\Omega_{1}$. The small correction is of the order of $10^{-6}$ with respect to the original level spacing.

\section{B. Quantum (anti-)Zeno effect for general spectral distribution}

Afterward, we generally investigate the QAZE for different spectral structures. Especially, we discover the condition when the QAZE disappears. In general, the interacting spectra are classified as three categories. They can be written with a uniform spectrum function [20]

$$
G(\omega)=A \omega_{c}^{1-s} \omega^{s} e^{-\omega / \omega_{c}},
$$

where $A$ is a constant and $\omega_{c}$ is the cutoff frequency. For an Ohmic spectrum, $s=1$ while $s<1$ and $s>1$ for sub-Ohmic and super-Ohmic spectra, respectively. In Fig. 4, the transition from the QAZE to the QZE is again observed. It is a predictable result since the peak of the spectrum function is located at

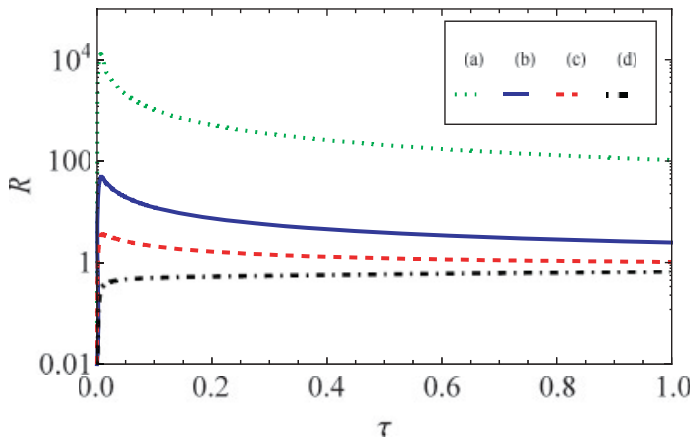

FIG. 4. (Color online) The decay rate vs measurement interval $\tau$ for different spectra. Here the dotted green line (a) is for super-Ohmic spectrum $s=2$, the solid blue line (b) is for Ohmic spectrum $s=1$, the dashed red line (c) is for sub-Ohmic spectrum $s=0.5$, and the dot-dashed black line (d) is for sub-Ohmic spectrum $s=0.002$, which satisfies Eq. (21). For all spectra we set $A=10^{-8}, \omega_{c} / \Omega=500$.

$\omega=s \omega_{c}$. As long as $s \omega_{c} \gg \Omega$, the QAZE definitely occurs. Moreover, on condition that

$$
\Omega_{1} \simeq \Omega=s \omega_{c},
$$

the QAZE is wiped out and only the QZE takes place, as shown by the dot-dashed black line in Fig. 4. Additionally, the difference between the decay rates with and without the RWA is plotted in Fig. 5. Since the contribution is no more than $10^{-3}$ for the given parameters $A=10^{-8}$ and $\omega_{c} / \Omega=500$, the counter-rotating terms thus can be neglected as the routine work done in quantum optics.

However, the preceding analysis is based on the assumption of a small energy-level shift. For some physical systems, this shift may play an important role in the existence of the QAZE. For a given interacting spectrum as shown in Eq. (20), the modified energy level spacing reads

$$
\Omega_{1}=\Omega+A e^{\frac{\Omega}{\omega_{c}}} \Omega^{s} \Gamma(1+s, 0) \Gamma\left(-s, \frac{\Omega}{\omega_{c}}\right),
$$

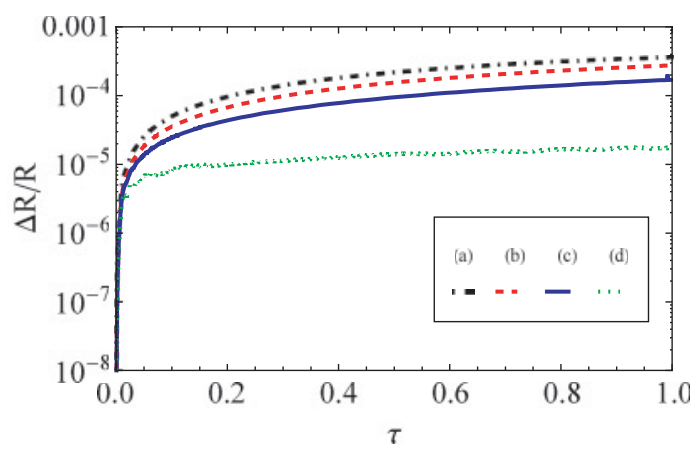

FIG. 5. (Color online) The decay rate difference $\Delta R=\mid R-$ $R_{\text {rwa }} \mid$ vs measurement interval $\tau$ for different spectra. Here the dot-dashed black line (a) is for sub-Ohmic spectrum $s=0.002$ with $\left|\Omega_{1}-\Omega\right| / \Omega=1.55 \times 10^{-3}$, the dashed red line (b) is for sub-Ohmic spectrum $s=0.5$ with $\left|\Omega_{1}-\Omega\right| / \Omega=9.41 \times 10^{-4}$, the solid blue line (c) is for Ohmic spectrum $s=1$ with $\left|\Omega_{1}-\Omega\right| / \Omega=6.57 \times$ $10^{-4}$, and the dotted green line (d) is for super-Ohmic spectrum $s=2$ with $\left|\Omega_{1}-\Omega\right| / \Omega=4.00 \times 10^{-4}$. Parameters are the same as those given in Fig. 4. 


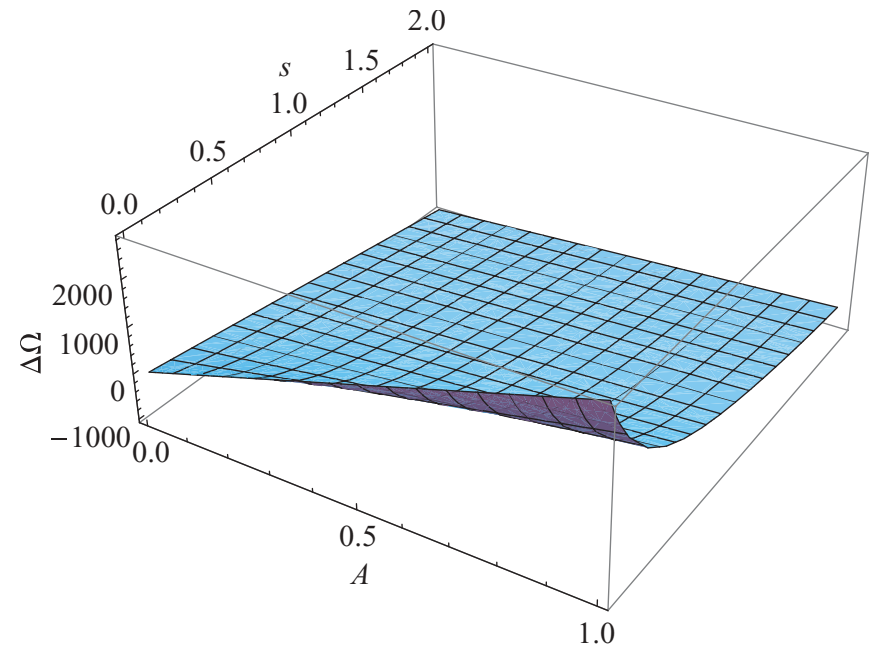

FIG. 6. (Color online) The frequency distance $\Delta \Omega=\Omega_{1}-s \omega_{c}$ vs the parameters $A$ and $s$ with $\omega_{c} / \Omega=500$.

where

$$
\Gamma(u, z)=\int_{z}^{\infty} t^{u-1} e^{-t} d t .
$$

As stated in the preceding, the QAZE disappears if the peaks of the measurement-induced atomic level broadening and the interacting spectrum coincide. Therefore, the distance $\Delta \Omega=\Omega_{1}-s \omega_{c}$ between these two peaks is plotted vs the parameters $A$ and $s$ in Fig. 6. As shown, the distance $\Delta \Omega$ increases monotonically with increasing $A$. This is because the energy-level shift, that is, the second term on the right-hand side of Eq. (22), is proportional to $A$. Physically speaking, the larger the parameter $A$ is, the stronger the coupling between the atom and the reservoir becomes. As a result of the stronger coupling, the energy-level shift is enlarged. Besides, it is seen that $\Delta \Omega$ falls as the parameter $s$ raises. Thus, for a matching pair of $A$ and $s$, the two peaks of $F\left(\omega, \Omega_{1}\right)$ and the interacting spectrum $G(\omega)$ are the same. In this case, there will be only the QZE. Besides, we also notice that the QZE was well explored for a two-level system in either a low- or high-frequency bath beyond the RWA [21].

\section{ALTERNATIVE APPROACH FOR THE DECAY PHENOMENON OF THE HYDROGEN ATOM}

In this section, with a different choice of the transformation $\exp \left(-S^{\prime}\right)$, we obtain the same result as the preceding section for the hydrogen atom. This transformation approach can also work well for other spectral distributions but here we do not repeat the straightforward calculations.

We choose the same transformation $U^{\prime}=\exp \left(-S^{\prime}\right)$ as that in Ref. [10], with

$$
S^{\prime}=\sum_{k} A_{k}\left[\left(b_{k}^{\dagger} \sigma^{+}-b_{k} \sigma^{-}\right)+\left(b_{k}^{\dagger} \sigma^{-}-b_{k} \sigma^{+}\right)\right]
$$

and

$$
A_{k}=\frac{-g_{k}}{\omega_{k}+\Omega}
$$

to eliminate the counter-rotating terms $b_{k}^{\dagger} \sigma^{+}+b_{k} \sigma^{-}$in the desired effective Hamiltonian. Although the preceding coefficients $A_{k}$ are the same as the ones in Eq. (5), this transformation is different from that in Eq. (3) in that it includes the slow-oscillating terms, that is, $b_{k}^{\dagger} \sigma^{-}-b_{k} \sigma^{+}$. As a consequence, it modifies not only the atomic energy level spacing, but also its coupling to the reservoir and thus the interacting spectrum.

By virtue of omitting higher order terms, that is, $b_{k}^{\dagger} b_{k^{\prime}}^{\dagger}, b_{k} b_{k^{\prime}}$, the effective Hamiltonian is straightforwardly given as

$$
H_{\mathrm{eff}}^{\prime}=\sum_{k} \omega_{k} b_{k}^{\dagger} b_{k}+\frac{\Omega^{\prime}}{2} \sigma_{z}+\sum_{k} g_{k}^{\prime}\left(b_{k} \sigma^{+}+\text {H.c. }\right),
$$

where the modified coupling constant is

$$
g_{k}^{\prime}=\frac{2 \Omega}{\omega_{k}+\Omega} g_{k}
$$

and the modified atomic energy level spacing is

$$
\Omega^{\prime}=\Omega+2 \sum_{k} \frac{\Omega g_{k} A_{k}}{\omega_{k}+\Omega} .
$$

We would like to mention that the term

$$
\sum_{k} \frac{\Omega g_{k} A_{k}}{\omega_{k}+\Omega}\left(1+b_{k}^{\dagger} b_{k}\right)
$$

is replaced with

$$
\sum_{k} \frac{\Omega g_{k} A_{k}}{\omega_{k}+\Omega}
$$

in the preceding calculation since their contribution results in small modification.

Since the original Hamiltonian $H$ and a state $|\psi(t)\rangle$ fulfill the Schrödinger equation $H|\psi(t)\rangle=i \partial_{t}|\psi(t)\rangle$, we emphasize that it is the transformed state $|\psi(t)\rangle^{S^{\prime}}=\exp \left(-S^{\prime}\right)|\psi(t)\rangle$ and the effective Hamiltonian $H_{\text {eff }}^{\prime}$ that meet the same requirement $H_{\mathrm{eff}}^{\prime}|\psi(t)\rangle^{S^{\prime}}=i \partial_{t}|\psi(t)\rangle^{S^{\prime}}$. Thus, in general cases the initial state $|\psi(0)\rangle$ before the transformation should be changed as $|\psi(0)\rangle^{S^{\prime}}=\exp \left(-S^{\prime}\right)|\psi(0)\rangle$ after the transformation.

In practice, the choice of the initial state relies on the concrete physical problem. As far as the vacuum-induced spontaneous decay is concerned, we should choose the bare excited state. We emphasize that this choice is consistent with the one in Ref. [8]. We also remark that it would be more reasonable to start from the same initial state when we refer to the influence of the counter-rotating terms on the QAZE. Besides, we can also choose the physical excited state elsewhere, that is, in Ref. [10]. It is a reasonable consideration since the ground state $|g,\{0\}\rangle$ of the Hamiltonian under RWA is replaced with $\exp \left(S^{\prime}\right)|g,\{0\}\rangle$ due to the presence of the counter-rotating terms in the interaction Hamiltonian (2) [22]. Therefore, the initial state may be $\exp \left(S^{\prime}\right)|e,\{0\}\rangle$ instead of $|e,\{0\}\rangle$ under the condition that the initial state is prepared from the ground state $\exp \left(S^{\prime}\right)|g,\{0\}\rangle$ through excitation by laser. These two different choices result in distinct consequences.

The problem is solved in the interaction picture with respect to

$$
U_{0}=e^{-i H_{0}^{\prime} t},
$$


with the "renormalized" free Hamiltonian

$$
H_{0}^{\prime}=\sum_{k} \omega_{k} b_{k}^{\dagger} b_{k}+\frac{\Omega^{\prime}}{2} \sigma_{z} .
$$

The interaction Hamiltonian $H_{1}^{I}=U_{0} H_{1}^{\prime} U_{0}^{+} \equiv U_{0}\left(H_{\mathrm{eff}}^{\prime}\right.$ $\left.H_{0}^{\prime}\right) U_{0}^{+}$reads

$$
H_{1}^{I}=\sum_{k} g_{k}^{\prime}\left[b_{k} \sigma^{+} e^{i\left(\Omega^{\prime}-\omega_{k}\right) t}+b_{k}^{\dagger} \sigma^{-} e^{-i\left(\Omega^{\prime}-\omega_{k}\right) t}\right] .
$$

The time evolution of the wave function

$$
\left|\psi_{I}^{\prime}(t)\right\rangle=\alpha(t)|e,\{0\}\rangle+\sum_{k} \beta_{k}(t)\left|g, 1_{k}\right\rangle
$$

is governed by the effective Hamiltonian $H_{1}^{I}$,

$$
i \partial_{t}\left|\psi_{I}^{\prime}\right\rangle=H_{1}^{I}\left|\psi_{I}^{\prime}\right\rangle
$$

Here $\left|g, 1_{k}\right\rangle$ denotes the atom in the ground state $|g\rangle$ and one excitation in the $k$ th mode.

Then, the coefficients meet the following demands

$$
\begin{gathered}
\dot{\alpha}=-i \sum_{k} g_{k}^{\prime} \beta_{k} e^{i\left(\Omega^{\prime}-\omega_{k}\right) t}, \\
\dot{\beta}_{k}=-i g_{k}^{\prime} \alpha e^{-i\left(\Omega^{\prime}-\omega_{k}\right) t} .
\end{gathered}
$$

However, when calculating the survival probability, we should return to the Schrödinger picture, that is,

$$
\begin{gathered}
\alpha \rightarrow \alpha e^{-i \frac{\Omega^{\prime}}{2} t}, \\
\beta_{k} \rightarrow \beta_{k} e^{-i\left(\omega_{k}-\frac{\Omega^{\prime}}{2}\right) t} .
\end{gathered}
$$

We remark that with the initial state $|e,\{0\}\rangle$, the considered survival probability for the excited state of the atom under the original Hamiltonian $H$ is

$$
\begin{aligned}
P(t) & =\operatorname{Tr}\left(|e\rangle\left\langle e\left|e^{-i H t}\right| e,\{0\}\right\rangle\langle e,\{0\}| e^{i H t}\right) \\
& =|x(t)|^{2} .
\end{aligned}
$$

Correspondingly, the effective Hamiltonian and the initial state after the preceding transformation are $H_{\mathrm{eff}}^{\prime}$ and $e^{-S^{\prime}}|e,\{0\}\rangle$, respectively. Then, one has the survival probability amplitude

$$
\begin{aligned}
x(t)= & \left\langle e,\{0\}\left|e^{S^{\prime}} e^{-i H_{\mathrm{eff}}^{\prime} t} e^{-S^{\prime}}\right| e,\{0\}\right\rangle \\
\simeq & C_{1}\left\langle e,\{0\}\left|e^{-i H_{\mathrm{eff}}^{\prime} t}\right| e,\{0\}\right\rangle \\
& -C_{2} \sum_{k} A_{k}\left\langle e,\{0\}\left|e^{-i H_{\mathrm{eff}}^{\prime} t}\right| g, 1_{k}\right\rangle \\
& -C_{2} \sum_{k} A_{k}\left\langle g, 1_{k}\left|e^{-i H_{\mathrm{eff}}^{\prime} t}\right| e,\{0\}\right\rangle \\
& +\sum_{k} A_{k}^{2}\left\langle g, 1_{k}\left|e^{-i H_{\mathrm{eff}}^{\prime} t}\right| g, 1_{k}\right\rangle,
\end{aligned}
$$

where we have dropped the off-diagonal terms for the fourth term on the right-hand side and

$$
\begin{gathered}
C_{1}=\left(1-\frac{1}{2} \sum_{k} A_{k}^{2}\right)^{2} \simeq 1-\sum_{k} A_{k}^{2}, \\
C_{2}=1-\frac{1}{2} \sum_{k} A_{k}^{2} .
\end{gathered}
$$

In Appendix A, we prove that the survival probability for the atom in the excited state is the same as the one for the initial state, namely, the first line in Eq. (37). After a series of deductions, the survival probability after $n$ measurements is written as

$$
P(t=n \tau)=|x(\tau)|^{2 n}=e^{-R t},
$$

where the decay rate

$$
R=2 \pi \int_{-\infty}^{\infty} d \omega F\left(\omega, \Omega^{\prime}\right) G^{\prime}(\omega)
$$

is the overlap integral of the measurement-induced atomic level broadening

$$
F\left(\omega, \Omega^{\prime}\right)=\frac{\tau}{2 \pi} \operatorname{sinc}^{2}\left(\frac{\omega-\Omega^{\prime}}{2} \tau\right)
$$

and the interacting spectrum

$$
G^{\prime}(\omega)=\sum_{k} f\left(\omega_{k}\right) g_{k}^{2} \delta\left(\omega-\omega_{k}\right)
$$

with

$$
f\left(\omega_{k}\right)=1+\frac{\left(3 \Omega-\Omega^{\prime}+2 \omega_{k}\right)\left(\Omega-\Omega^{\prime}\right)}{\left(\omega_{k}+\Omega\right)^{2}} .
$$

Notice that the measurement-induced atomic level broadening differs from ours in Eq. (14) in that it is centered at a different modified level spacing $\Omega^{\prime}$. The interacting spectrum is also altered with an additional factor $f(\omega)$, contrary to the unaltered one in Eq. (15). For the necessary details, please refer to Appendix B.

For the hydrogen atom, the modified interacting spectrum of the $2 p-1 s$ transition is

$$
G_{2 p-1 s}^{\prime}(\omega)=f(\omega) \frac{\eta \omega}{\left[1+\left(\frac{\omega}{\omega_{c}}\right)^{2}\right]^{4}},
$$

with $\eta$ and $\omega_{c}$ already given in Eq. (18). Notice that the second term in Eq. (44) is a small correction to the one with the RWA of the order of $10^{-8}$. This is in consistence with the orders of the numerical results of $\Delta R$ in Figs. 7 and 8.

The relation between the decay rate $R$ and the measurement interval $\tau$ is plotted in Fig. 7. As shown in this figure, the

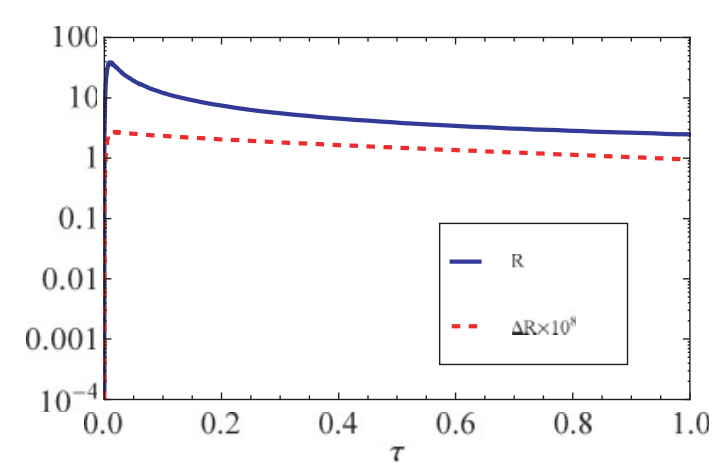

FIG. 7. (Color online) The decay rate vs measurement interval $\tau$ for the $2 p-1 s$ transition of the hydrogen atom. Here the solid blue line is for $R$ and the dashed red line is for $\Delta R=\left|R-R_{\mathrm{rwa}}\right| \cdot \mid \Omega^{\prime}-$ $\Omega \mid / \Omega=5.69 \times 10^{-8}$ and $\omega_{c} / \Omega=550$. Notice that $\Delta R$ is enlarged $10^{8}$ times. 


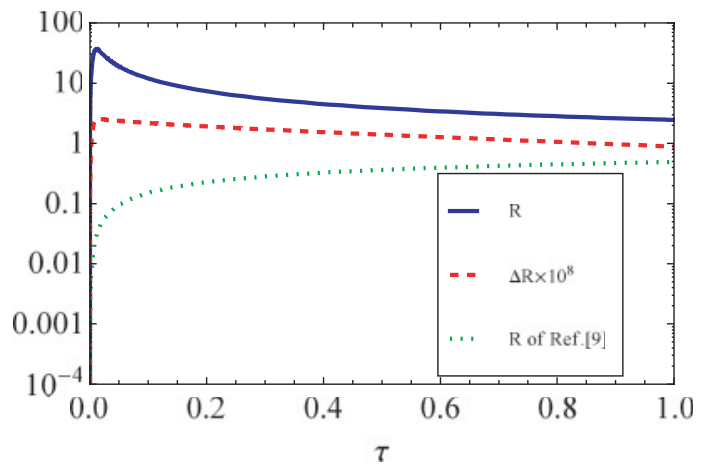

FIG. 8. (Color online) The decay rate vs measurement interval $\tau$ for the $3 p-1 s$ transition of the hydrogen atom. Here the solid blue line is for $R$, the dashed red line is for $\Delta R=\left|R-R_{\mathrm{rwa}}\right|$, and the dotted green line is for the result from Ref. [10]. $\left|\Omega^{\prime}-\Omega\right| / \Omega=5.32 \times 10^{-8}$ and $\omega_{c} / \Omega=412$. Notice that $\Delta R$ is enlarged $10^{8}$ times.

decay rate is well separated into two parts with the climax being the boundary. In the left part, as the measurements are done more and more frequently, that is, $\tau \rightarrow 0$, the decay rate falls monotonously. When it is less than the decay rate without measurement

$$
R_{0}^{\prime}=2 \pi G^{\prime}\left(\Omega^{\prime}\right),
$$

that is, $R / R_{0}^{\prime}<1$, the QZE takes place. To the right of the climax, the opposite trend is witnessed. In this region, the shorter the measurement interval is, the larger the decay rate is. Since $R / R_{0}^{\prime}>1$ for the whole region to the right of the climax, one can observe the QAZE, which was predicted to be obliterated due to the counter-rotating terms [10]. The discrepancy between their result and ours is attributed to the different choices of the initial states [10]. Here we also emphasize that our approach is very simple and concise in contrast to theirs, which is shown in Appendix B.

For more evidence, we resort to the $3 p-1 s$ transition of the hydrogen atom. The interacting spectrum is adjusted as

$$
G_{3 p-1 s}^{\prime}(\omega)=f(\omega) \frac{\eta^{\prime} \omega\left[1+2\left(\frac{\omega}{\omega_{c}^{\prime}}\right)^{2}\right]^{2}}{\left[1+\left(\frac{\omega}{\omega_{c}^{\prime}}\right)^{2}\right]^{6}}
$$

where $\eta^{\prime}$ and $\omega_{c}^{\prime}$ are given in Eq. (19). The result of the numerical calculations is displayed in Fig. 8. Here we again observe the complete opposite predictions of the QAZE, contrary to Ref. [10]. Moreover, the RWA offers a good approximation in the weak-coupling limit since the disagreement between its and the exact result is trivial.

\section{CONCLUSION}

In summary, we have studied the role of the counter-rotating terms of the atomic couplings to the reservoir in the irreversible atomic transition during the repetitive measurements. By the generalized Fröhlich-Nakajima transformation, the exactly solvable Hamiltonian is effectively obtained without the RWA in the form of the standard "spin-boson" model. We discovered that when we consider the spontaneous decay of the bare excited state even without the RWA, the QAZE remains if the proper measurement interval is given. We also saw a transition from the QZE to the QAZE as the measurement interval changes. As for the findings in Ref. [10] and its following articles, it is observed that the disappearance of the QAZE under the approach without the RWA is mainly due to the choice of the physical excited state. In Ref. [10], this initial state is the excited state of the renormalized Hamiltonian, which is essentially an entangled state of photons and atomic states. For the physical systems in a realistic world, the influence of the weak-coupling counter-rotating terms on the decay rate is very small and can be negligible. We have utilized two different approaches for the generalization of the Fröhlich-Nakajima transformation. For the same initial state, that is, the bare excited state, consistent conclusion is obtained for the interacting spectra of the hydrogen atom. By comparing the two effective Hamiltonians, we find out that in our approach there is only one parameter modified, contrary to one more set in Ref. [10]. To further verify the universality of the presence of the QAZE, we also extend our investigation to different types of spectra. It is discovered that when the cutoff frequency and the atomic level spacing fulfill some condition, only the QZE emerges for the sub-Ohmic spectrum. We notice that by means of the QZE in the dynamic version a quantum switch was proposed to control the transport of a single photon in a one-dimensional waveguide under the RWA [23]. However, we might look forward to some novel features if no RWA is invoked.

Besides, it is worth underlining that the choice of the different initial states depends on the specific physical problem. So far as the QAZE for the vacuum-induced spontaneous decay is concerned, we should choose the bare excited state in that it is the vacuum that induces the spontaneous decay of the atomic excitation. On the other hand, on account of the preparation of the initial state, the physical excited state might be a better choice as well because it can be feasibly excited from the ground state of the original Hamiltonian.

\section{ACKNOWLEDGMENTS}

This work was supported by NSFC through Grants No. 10974209 and No. 10935010 and by the National 973 program (Grant No. 2006CB921205).

\section{APPENDIX A: SURVIVAL PROBABILITY $P(t)$}

When we refer to the QZE and QAZE, we take a projective measurement on the atom. Thus, we shall trace over all the possible states of the fields. Based on the preceding considerations, we give the detailed deduction about the survival probability $P(t)=|x(t)|^{2}$.

Before the transformation, the original Hamiltonian is

$$
H=\sum_{k} \omega_{k} b_{k}^{\dagger} b_{k}+\frac{\Omega}{2} \sigma_{z}+\sum_{k} g_{k}\left(b_{k}+b_{k}^{\dagger}\right)\left(\sigma^{+}+\sigma^{-}\right)
$$

with the chosen initial state to be

$$
|\Psi(0)\rangle=|e,\{0\}\rangle .
$$

Then, we take a unitary transformation $e^{-S}$ with

$$
S=\sum_{k} A_{k}\left(b_{k}^{\dagger} \sigma^{+}-b_{k} \sigma^{-}\right)
$$


and the Hamiltonian is approximated to the second order as

$$
\begin{aligned}
H_{\text {eff }} & =e^{-S} H e^{S} \\
& =\sum_{k} \omega_{k} b_{k}^{\dagger} b_{k}+\frac{\Omega_{1}}{2} \sigma_{z}+\sum_{k} g_{k}\left(b_{k} \sigma^{+}+\text {H.c. }\right),
\end{aligned}
$$

in company with a transformed initial state

$$
\begin{aligned}
|\Psi(0)\rangle^{S} & =e^{-S}|\Psi(0)\rangle \\
& =e^{-S}|e,\{0\}\rangle \\
& =|e,\{0\}\rangle .
\end{aligned}
$$

As a result, the evolution of the state reads

$$
|\Psi(t)\rangle^{S}=e^{-i H_{\mathrm{eff}} t}|\Psi(0)\rangle^{S} .
$$

When calculating the survival probability for the atom in the excited state, we shall return to the original picture and the density matrix for the total system is straightforwardly given as

$$
\begin{aligned}
\rho(t) & =|\Psi(t)\rangle\langle\Psi(t)| \\
& =e^{S}|\Psi(t)\rangle^{S S}\langle\Psi(t)| e^{-S} \\
& =e^{S} e^{-i H_{\mathrm{eff}} t} e^{-S}|\Psi(0)\rangle\langle\Psi(0)| e^{S} e^{i H_{\mathrm{eff}} t} e^{-S} \\
& =e^{S} e^{-i H_{\mathrm{eff}} t} e^{-S}|e,\{0\}\rangle\langle e,\{0\}| e^{S} e^{i H_{\mathrm{eff}} t} e^{-S} .
\end{aligned}
$$

The reduced density matrix for the atom is traced over the degrees of fields, that is,

$$
\begin{aligned}
\rho_{s}(t)= & \operatorname{Tr}_{B}|\Psi(t)\rangle\langle\Psi(t)| \\
= & \left\langle\{0\}\left|e^{S} e^{-i H_{\mathrm{eff}} t} e^{-S}\right| e,\{0\}\right\rangle\left\langle e,\{0\}\left|e^{S} e^{i H_{\mathrm{eff}} t} e^{-S}\right|\{0\}\right\rangle \\
& +\sum_{k}\left\langle 1_{k}\left|e^{S} e^{-i H_{\mathrm{eff}} t} e^{-S}\right| e,\{0\}\right\rangle\left\langle e,\{0\}\left|e^{S} e^{i H_{\mathrm{eff}} t} e^{-S}\right| 1_{k}\right\rangle \\
& +\sum_{k, k^{\prime}}\left\langle 1_{k} 1_{k^{\prime}}\left|e^{S} e^{-i H_{\mathrm{eff}} t} e^{-S}\right| e,\{0\}\right\rangle \\
& \times\left\langle e,\{0\}\left|e^{S} e^{i H_{\mathrm{eff}} t} e^{-S}\right| 1_{k} 1_{k^{\prime}}\right\rangle+\cdots .
\end{aligned}
$$

Therefore, the survival probability of the excited state of the atom is

$$
\begin{aligned}
\rho_{s}^{e e}(t)= & \operatorname{Tr}_{s}\left(|e\rangle\langle e| \rho_{s}(t)\right) \\
\simeq & \left\langle e,\{0\}\left|e^{S} e^{-i H_{\mathrm{eff}} t} e^{-S}\right| e,\{0\}\right\rangle\left\langle e,\{0\}\left|e^{S} e^{i H_{\mathrm{eff}} t} e^{-S}\right| e,\{0\}\right\rangle \\
& +\sum_{k}\left\langle e, 1_{k}\left|e^{S} e^{-i H_{\mathrm{eff}} t} e^{-S}\right| e,\{0\}\right\rangle \\
& \times\left\langle e,\{0\}\left|e^{S} e^{i H_{\mathrm{eff}} t} e^{-S}\right| e, 1_{k}\right\rangle \\
& +\sum_{k, k^{\prime}}\left\langle e, 1_{k} 1_{k^{\prime}}\left|e^{S} e^{-i H_{\mathrm{eff}} t} e^{-S}\right| e,\{0\}\right\rangle \\
& \times\left\langle e,\{0\}\left|e^{S} e^{i H_{\mathrm{eff}} t} e^{-S}\right| e, 1_{k} 1_{k^{\prime}}\right\rangle+\cdots .
\end{aligned}
$$

In the following deductions, we show that multiple excitation terms can be omitted as they lead to small corrections to the final result. For the case of two excitations,

$$
\begin{aligned}
e^{-S}\left|e, 1_{k}\right\rangle & \simeq\left(I-S+\frac{1}{2} S^{2}\right)\left|e, 1_{k}\right\rangle \\
& =\left|e, 1_{k}\right\rangle+A_{k}|g,\{0\}\rangle-\frac{1}{2} \sum_{k^{\prime}} A_{k} A_{k^{\prime}}\left|e, 1_{k^{\prime}}\right\rangle
\end{aligned}
$$

is a superposition of states with the total excitation of an even number, while

$$
\begin{aligned}
e^{-S}|e,\{0\}\rangle & \simeq\left(I-S+\frac{1}{2} S^{2}\right)|e,\{0\}\rangle \\
& =|e,\{0\}\rangle
\end{aligned}
$$

has only one excitation. On account of $H_{\text {eff's property of }}$ conserving the total number of excitation, the second term in Eq. (A9) vanishes.

For the case of three excitations,

$$
\begin{aligned}
& e^{-S}\left|e, 1_{k} 1_{k^{\prime}}\right\rangle \\
& \simeq\left(I-S+\frac{1}{2} S^{2}\right)\left|e, 1_{k} 1_{k^{\prime}}\right\rangle \\
& =\left|e, 1_{k} 1_{k^{\prime}}\right\rangle+A_{k}\left|g, 1_{k^{\prime}}\right\rangle+A_{k^{\prime}}\left|g, 1_{k}\right\rangle \\
& \quad-\frac{1}{2} \sum_{k^{\prime \prime}}\left(A_{k} A_{k^{\prime \prime}}\left|e, 1_{k^{\prime}} 1_{k^{\prime \prime}}\right\rangle+A_{k^{\prime}} A_{k^{\prime \prime}}\left|e, 1_{k} 1_{k^{\prime \prime}}\right\rangle\right) .
\end{aligned}
$$

Then, the third term on the right-hand side of Eq. (A9) equals

$$
\begin{aligned}
& \sum_{k, k^{\prime}}\left|\left\langle e, 1_{k} 1_{k^{\prime}}\left|e^{S} e^{-i H_{\mathrm{eff}} t} e^{-S}\right| e,\{0\}\right\rangle\right|^{2} \\
& \simeq 2 \sum_{k, k^{\prime}} A_{k}^{2}\left|\left\langle g, 1_{k^{\prime}}\left|e^{-i H_{\mathrm{eff}} t}\right| e,\{0\}\right\rangle\right|^{2} \\
& \quad+2\left|\sum_{k} A_{k}\left\langle g, 1_{k}\left|e^{-i H_{\mathrm{eff}} t}\right| e,\{0\}\right\rangle\right|^{2} .
\end{aligned}
$$

In the interaction picture, the interaction Hamiltonian reads

$$
H^{I}=\sum_{k} g_{k}\left[b_{k} \sigma^{+} e^{i\left(\Omega_{1}-\omega_{k}\right) t}+b_{k}^{\dagger} \sigma^{-} e^{-i\left(\Omega_{1}-\omega_{k}\right) t}\right] .
$$

The time evolution of the wave function,

$$
\left|\psi_{I}\right\rangle=\alpha(t)|e,\{0\}\rangle-\sum_{k} \beta_{k}(t)\left|g, 1_{k}\right\rangle,
$$

is governed by the Hamiltonian $H^{I}$,

$$
i \partial_{t}\left|\psi_{I}\right\rangle=H^{I}\left|\psi_{I}\right\rangle
$$

Straightforwardly, we attain the equations for the coefficients as

$$
\begin{gathered}
\dot{\alpha}=-i \sum_{k} g_{k} \beta_{k} e^{i\left(\Omega_{1}-\omega_{k}\right) t}, \\
\dot{\beta}_{k}=-i g_{k} \alpha e^{-i\left(\Omega_{1}-\omega_{k}\right) t} .
\end{gathered}
$$

The first term of Eq. (A13) is equivalent to $2 \sum_{k, k^{\prime}} A_{k}^{2}\left|\beta_{k^{\prime}}(t)\right|^{2}$ when $\alpha(0)=1$. We can formally integrate Eq. (A18) and replace $\alpha\left(t^{\prime}\right)$ with 1 to have

$$
\begin{aligned}
\beta_{k}(t) & =-i \int_{0}^{t} d t^{\prime} g_{k} \alpha\left(t^{\prime}\right) e^{-i\left(\Omega_{1}-\omega_{k}\right) t^{\prime}} \\
& \simeq-i \int_{0}^{t} d t^{\prime} g_{k} e^{-i\left(\Omega_{1}-\omega_{k}\right) t^{\prime}} \\
& =g_{k} \frac{e^{-i\left(\Omega_{1}-\omega_{k}\right) t}-1}{\Omega_{1}-\omega_{k}} \\
& =g_{k} \frac{-2 \sin ^{2} \frac{\left(\Omega_{1}-\omega_{k}\right) t}{2}-i \sin \left(\Omega_{1}-\omega_{k}\right) t}{\Omega_{1}-\omega_{k}} .
\end{aligned}
$$


As a result, the first term of Eq. (A13),

$$
\begin{aligned}
& 2 \sum_{k, k^{\prime}} A_{k}^{2}\left|\beta_{k^{\prime}}(t)\right|^{2} \\
& \quad=2 \sum_{k} A_{k}^{2} \sum_{k^{\prime}} g_{k^{\prime}}^{2} \frac{4 \sin ^{4} \frac{\left(\Omega_{1}-\omega_{k^{\prime}}\right) t}{2}+\sin ^{2}\left(\Omega_{1}-\omega_{k^{\prime}}\right) t}{\left(\Omega_{1}-\omega_{k^{\prime}}\right)^{2}},
\end{aligned}
$$

can be neglected based on the following considerations. For one thing, the first term in the second summation is proportional to $t^{4}$ in the short time limit and thus can be omitted. For another, the factor $\sum_{k} A_{k}^{2}$ is a small quantity, that is, typically of the order of $10^{-8}$ for the hydrogen atom.

Moreover, the second term on the right-hand side of Eq. (A13) is equivalent to $2\left|\sum_{k} A_{k} \beta_{k}(t) \exp \left(-i \omega_{k} t\right)\right|^{2}$, where $\alpha(0)=1$ and the factor $\exp \left(-i \omega_{k} t\right)$ is due to transformation back to the Schrödinger picture. Here

$$
\begin{aligned}
& 2\left|\sum_{k} A_{k} \beta_{k}(t) e^{-i \omega_{k} t}\right|^{2} \\
& =2\left|\sum_{k} A_{k} g_{k} \frac{-2 \sin ^{2} \frac{\left(\Omega_{1}-\omega_{k}\right) t}{2}-i \sin \left(\Omega_{1}-\omega_{k}\right) t}{\Omega_{1}-\omega_{k}}\right|^{2} \\
& =\frac{t^{4}}{2}\left[\int_{-\infty}^{\infty} d \omega \operatorname{sinc}^{2} \frac{\left(\Omega_{1}-\omega\right) t}{2} \sum_{k} \frac{\Omega_{1}-\omega}{\Omega_{1}+\omega} g_{k}^{2} \delta\left(\omega-\omega_{k}\right)\right]^{2} \\
& \quad+2 t^{2}\left[\int_{-\infty}^{\infty} d \omega \operatorname{sinc}\left(\Omega_{1}-\omega\right) t \sum_{k} \frac{g_{k}^{2} \delta\left(\omega-\omega_{k}\right)}{\Omega_{1}+\omega}\right]^{2},
\end{aligned}
$$

where the first term is proportional to $t^{4}$ in the short time limit, and the second term is of higher order with respect to the first term in Eq. (A9).

As a consequence, the second and third terms on the righthand side of Eq. (A9) can be neglected. In other words, the contributions from the multiple-excitation terms result in a small correction to the final result, and thus we have

$$
\rho_{s}^{e e}(t)=\left|\left\langle e,\{0\}\left|e^{S} e^{-i H_{\mathrm{eff}} t} e^{-S}\right| e,\{0\}\right\rangle\right|^{2},
$$

which is exactly the same as the one in Eq. (12) for a single measurement.

For the second approach in Sec. IV with

$$
S^{\prime}=\sum_{k} A_{k}\left[\left(b_{k}^{\dagger} \sigma^{+}-b_{k} \sigma^{-}\right)+\left(b_{k}^{\dagger} \sigma^{-}-b_{k} \sigma^{+}\right)\right]
$$

we still have Eq. (A9),

$$
\begin{aligned}
\rho_{s}^{e e}(t)= & \operatorname{Tr}_{s}\left(|e\rangle\langle e| \rho_{s}(t)\right) \\
\simeq & \left\langle e,\{0\}\left|e^{S^{\prime}} e^{-i H_{\text {eff }}^{\prime} e^{-S^{\prime}}}\right| e,\{0\}\right\rangle \\
& \times\left\langle e,\{0\}\left|e^{S^{\prime}} e^{i H_{\text {eff }}^{\prime} t} e^{-S^{\prime}}\right| e,\{0\}\right\rangle \\
& +\sum_{k}\left\langle e, 1_{k}\left|e^{S^{\prime}} e^{-i H_{\text {eff }}^{\prime} t} e^{-S^{\prime}}\right| e,\{0\}\right\rangle \\
& \times\left\langle e,\{0\}\left|e^{S^{\prime}} e^{i H_{\text {eff }} t} e^{-S^{\prime}}\right| e, 1_{k}\right\rangle \\
& +\sum_{k, k^{\prime}}\left\langle e, 1_{k} 1_{k^{\prime}}\left|e^{S^{\prime}} e^{-i H_{\text {eff }}^{\prime} t} e^{-S^{\prime}}\right| e,\{0\}\right\rangle \\
& \times\left\langle e,\{0\}\left|e^{S^{\prime}} e^{i H_{\text {eff }}^{\prime} t} e^{-S^{\prime}}\right| e, 1_{k} 1_{k^{\prime}}\right\rangle+\cdots
\end{aligned}
$$

In this case, the effective Hamiltonian is replaced with

$$
\begin{aligned}
H_{\text {eff }}^{\prime} & =e^{-S^{\prime}} H e^{S^{\prime}} \\
& =\sum_{k} \omega_{k} b_{k}^{\dagger} b_{k}+\frac{\Omega^{\prime}}{2} \sigma_{z}+\sum_{k} g_{k}^{\prime}\left(b_{k} \sigma^{+}+\text {H.c. }\right),
\end{aligned}
$$

while the transformed initial state is given as

$$
\begin{aligned}
|\Psi(0)\rangle^{S^{\prime}}= & e^{-S^{\prime}}|\Psi(0)\rangle \\
= & e^{-S^{\prime}}|e,\{0\}\rangle \\
\simeq & {\left[I-S^{\prime}+\frac{1}{2}\left(S^{\prime}\right)^{2}\right]|e,\{0\}\rangle } \\
= & \left(1-\frac{1}{2} \sum_{k} A_{k}^{2}\right)|e,\{0\}\rangle-\sum_{k} A_{k}\left|g, 1_{k}\right\rangle \\
& +\frac{1}{2} \sum_{k, k^{\prime}} A_{k} A_{k^{\prime}}\left|e, 1_{k} 1_{k^{\prime}}\right\rangle .
\end{aligned}
$$

Then we obtain the transformed state at time $t$,

$$
\left|\Psi^{\prime}(t)\right\rangle^{S^{\prime}}=e^{-i H_{\text {eff }}^{\prime} t}\left|\Psi^{\prime}(0)\right\rangle^{S^{\prime}} .
$$

As

$$
\begin{aligned}
e^{-S^{\prime}}\left|e, 1_{k}\right\rangle \simeq & {\left[I-S^{\prime}+\frac{1}{2}\left(S^{\prime}\right)^{2}\right]\left|e, 1_{k}\right\rangle } \\
= & \left(1-\frac{1}{2} \sum_{k^{\prime}} A_{k^{\prime}}^{2}\right)\left|e, 1_{k}\right\rangle+\sum_{k} A_{k}|g,\{0\}\rangle \\
& -\sum_{k} A_{k^{\prime}}\left|g, 1_{k} 1_{k^{\prime}}\right\rangle-\sum_{k^{\prime}} A_{k} A_{k^{\prime}}\left|e, 1_{k^{\prime}}\right\rangle \\
& +\frac{1}{2} \sum_{k^{\prime}, k^{\prime \prime}} A_{k^{\prime}} A_{k^{\prime \prime}}\left|e, 1_{k} 1_{k^{\prime}} 1_{k^{\prime \prime}}\right\rangle
\end{aligned}
$$

and

$$
\begin{aligned}
e^{-S^{\prime}}|e,\{0\}\rangle \simeq & {\left[I-S^{\prime}+\frac{1}{2}\left(S^{\prime}\right)^{2}\right]|e,\{0\}\rangle } \\
= & \left(1-\frac{1}{2} \sum_{k^{\prime}} A_{k^{\prime}}^{2}\right)|e, 0\rangle-\sum_{k} A_{k}\left|g, 1_{k}\right\rangle \\
& +\frac{1}{2} \sum_{k^{\prime}, k} A_{k^{\prime}} A_{k}\left|e, 1_{k} 1_{k^{\prime}}\right\rangle
\end{aligned}
$$

are of even and odd numbers of total excitations, respectively, we have a vanishing second term on the right-hand side of Eq. (A23).

Because the contribution from

$$
\begin{aligned}
e^{-S^{\prime}}\left|e, 1_{k} 1_{k^{\prime}}\right\rangle & \\
\simeq & {\left[I-S^{\prime}+\frac{1}{2}\left(S^{\prime}\right)^{2}\right]\left|e, 1_{k} 1_{k^{\prime}}\right\rangle } \\
= & \left|e, 1_{k} 1_{k^{\prime}}\right\rangle+A_{k}\left|g, 1_{k^{\prime}}\right\rangle+A_{k^{\prime}}\left|g, 1_{k}\right\rangle \\
& \quad-\sum_{k^{\prime \prime}} A_{k^{\prime \prime}}\left|g, 1_{k} 1_{k^{\prime}} 1_{k^{\prime \prime}}\right\rangle-\frac{1}{2} \sum_{k^{\prime \prime}} A_{k} A_{k^{\prime \prime}}\left|e, 1_{k^{\prime}} 1_{k^{\prime \prime}}\right\rangle \\
& +A_{k} A_{k^{\prime}}|e, 0\rangle+\frac{1}{2} \sum_{k^{\prime \prime}, k^{\prime \prime \prime}} A_{k^{\prime \prime}} A_{k^{\prime \prime \prime}}\left|e, 1_{k} 1_{k^{\prime}} 1_{k^{\prime \prime}} 1_{k^{\prime \prime \prime}}\right\rangle
\end{aligned}
$$




$$
\begin{aligned}
& -\frac{1}{2} \sum_{k^{\prime \prime}} A_{k} A_{k^{\prime \prime}}\left|e, 1_{k^{\prime}} 1_{k^{\prime \prime}}\right\rangle-\frac{1}{2} \sum_{k^{\prime \prime}} A_{k^{\prime}} A_{k^{\prime \prime}}\left|e, 1_{k} 1_{k^{\prime \prime}}\right\rangle \\
& -\frac{1}{2} \sum_{k^{\prime \prime}} A_{k^{\prime}} A_{k^{\prime \prime}}\left|e, 1_{k} 1_{k^{\prime \prime}}\right\rangle-\frac{1}{2} \sum_{k^{\prime \prime}} A_{k^{\prime \prime}}^{2}\left|e, 1_{k} 1_{k^{\prime}}\right\rangle
\end{aligned}
$$

is of an order higher than $A_{k}^{2}$, we can further omit the third term of Eq. (A23).

In total, we can neglect all nonzero-photon terms in Eq. (A23). Thus, based on the preceding calculations, we still have

$$
\rho_{s}^{e e}(t)=|x(t)|^{2}=\left|\left\langle e,\{0\}\left|e^{S^{\prime}} e^{-i H_{\mathrm{eff}}^{\prime} t} e^{-S^{\prime}}\right| e,\{0\}\right\rangle\right|^{2}
$$

for the second approach, where the survival probability $x(t)$ is the same as Eq. (37).

Judging from the preceding reductions, we may safely arrive at the conclusion that for both cases the survival probability of the atom in the excited state coincides with the survival probability of the initial state $|e,\{0\}\rangle$ and the single excitation approximation is reasonable.

\section{APPENDIX B: SURVIVAL AMPLITUDE $x(t)$ FOR THE SECOND APPROACH}

As shown in Eq. (37), the survival probability $x(t)$ is the summation of four terms due the modified initial state after the transformation. Since the calculation of the first term in Eq. (37) was already shown elsewhere, that is, Ref. [8], we offer the detailed calculation of the remaining parts in addition to the first term.

For convenience, we multiply $x(t)$ [Eq. (37)] by a factor $\exp \left(i \Omega^{\prime} t / 2\right)$ and have

$$
\begin{aligned}
x(t) e^{i \frac{\Omega^{\prime}}{2} t}= & C_{1}\left\langle e,\{0\}\left|e^{-i H_{\text {eff }} t}\right| e,\{0\}\right\rangle e^{i \frac{\Omega^{\prime}}{2} t} \\
& -C_{2} \sum_{k} A_{k}\left\langle e,\{0\}\left|e^{-i H_{\text {eff }} t}\right| g, 1_{k}\right\rangle e^{i \frac{\Omega^{\prime}}{2} t} \\
& -C_{2} \sum_{k} A_{k}\left\langle g, 1_{k}\left|e^{-i H_{\text {eff }} t}\right| e,\{0\}\right\rangle e^{i \frac{\Omega^{\prime}}{2} t} \\
& +\sum_{k} A_{k}^{2}\left\langle g, 1_{k}\left|e^{-i H_{\text {eff }} t}\right| g, 1_{k}\right\rangle e^{i \frac{\Omega^{\prime}}{2} t}
\end{aligned}
$$

On the right-hand side of the preceding equation, the first term

$$
\left\langle e,\{0\}\left|e^{-i H_{\mathrm{eff}} t}\right| e,\{0\}\right\rangle
$$

is equivalent to $\alpha(t)$ when $\alpha(0)=1$. Equation (35) can be formally integrated to yield

$$
\beta_{k}=-i \int_{0}^{t} d t^{\prime} g_{k}^{\prime} \alpha e^{-i\left(\Omega^{\prime}-\omega_{k}\right) t^{\prime}}
$$

By substituting it into Eq. (34), we have

$$
\dot{\alpha}=-\sum_{k} \int_{0}^{t} d t^{\prime}\left(g_{k}^{\prime}\right)^{2} \alpha e^{-i\left(\Omega^{\prime}-\omega_{k}\right) t^{\prime}} e^{i\left(\Omega^{\prime}-\omega_{k}\right) t}
$$

For a sufficient short time $t$, we can replace $\alpha\left(t_{2}\right)$ with $\alpha(0)=1$ and thus

$$
\begin{aligned}
\alpha(t) & \simeq 1-\int_{0}^{t} d t_{1} \int_{0}^{t_{1}} d t_{2} \sum_{k}\left(g_{k}^{\prime}\right)^{2} e^{i\left(\Omega^{\prime}-\omega_{k}\right)\left(t_{1}-t_{2}\right)} \\
& =1-t \int_{0}^{t} d t^{\prime}\left(1-\frac{t^{\prime}}{t}\right) e^{i \Omega^{\prime} t^{\prime}} \sum_{k}\left(g_{k}^{\prime}\right)^{2} e^{-i \omega_{k} t^{\prime}} \\
& =1-I_{\alpha}(t) .
\end{aligned}
$$

When transforming it back to the Schrödinger picture, we have

$$
\left\langle e,\{0\}\left|e^{-i H_{\text {eff }} t}\right| e,\{0\}\right\rangle=\alpha e^{-i \frac{\Omega^{\prime}}{2} t} .
$$

By multiplying a factor $\exp \left(i \Omega^{\prime} t / 2\right)$, the time-dependent factor is canceled,

$$
\left\langle e,\{0\}\left|e^{-i H_{\mathrm{eff}} t}\right| e,\{0\}\right\rangle e^{i \frac{\Omega^{\prime}}{2} t}=\alpha .
$$

The following function is used in the calculation of $x(t)$ :

$$
\begin{aligned}
2 \operatorname{Re} I_{\alpha}(t) & =2 \operatorname{Re}\left[t \int_{0}^{t} d t^{\prime}\left(1-\frac{t^{\prime}}{t}\right) e^{i \Omega^{\prime} t^{\prime}} \sum_{k}\left(g_{k}^{\prime}\right)^{2} e^{-i \omega_{k} t^{\prime}}\right] \\
& =2 \pi t \int_{-\infty}^{\infty} d \omega F\left(\omega, \Omega^{\prime}\right) G_{1}(\omega),
\end{aligned}
$$

which is the overlap integral of the measurement function

$$
\begin{aligned}
F\left(\omega, \Omega^{\prime}\right) & =\frac{1}{2 \pi} \int_{-\infty}^{\infty} d t^{\prime}\left(1-\frac{\left|t^{\prime}\right|}{t}\right) e^{i \Omega^{\prime} t^{\prime}} \theta\left(t-\left|t^{\prime}\right|\right) e^{-i \omega t^{\prime}} \\
& =\frac{t}{2 \pi} \operatorname{sinc}^{2}\left(\frac{\omega-\Omega^{\prime}}{2} t\right)
\end{aligned}
$$

and the interacting spectrum

$$
\begin{aligned}
G_{1}(\omega) & =\frac{1}{2 \pi} \int_{-\infty}^{\infty} d t^{\prime} \sum_{k}\left(g_{k}^{\prime}\right)^{2} e^{-i \omega_{k} t^{\prime}} e^{i \omega t^{\prime}} \\
& =\sum_{k}\left(g_{k}^{\prime}\right)^{2} \delta\left(\omega-\omega_{k}\right) .
\end{aligned}
$$

Before calculating the second and third terms in Eq. (B1), we prove these two terms to be equal to simplify the calculations. For a general Hamiltonian $H$, which is time-independent and satisfies $H_{a b} \equiv\langle a|H| b\rangle=H_{b a}$ for any two states $|a\rangle$ and $|b\rangle$ in the complete Hilbert space, one has

$$
\begin{aligned}
F_{a b} & =\left\langle a\left|e^{-i H t}\right| b\right\rangle \\
& =\left\langle a\left|\sum_{n} \frac{(-i t H)^{n}}{n !}\right| b\right\rangle \\
& =\left\langle a\left|\sum_{n} \frac{(-i t H)^{2 n}}{(2 n) !}\right| b\right\rangle+\left\langle a\left|\sum_{n} \frac{(-i t H)^{2 n+1}}{(2 n+1) !}\right| b\right\rangle \\
& \equiv \operatorname{Re}\left(F_{a b}\right)+i \operatorname{Im}\left(F_{a b}\right) .
\end{aligned}
$$

It is obvious that the first term on the right-hand side of the preceding equation is real and the second term is pure imaginary. On the other hand, one has

$$
\begin{aligned}
& \left\langle b\left|e^{-i H t}\right| a\right\rangle \\
& =\left[\left(\left\langle b\left|e^{-i H t}\right| a\right\rangle\right)^{\dagger}\right]^{\dagger} \\
& =\left[\left\langle a\left|e^{i H t}\right| b\right\rangle\right]^{\dagger}
\end{aligned}
$$




$$
\begin{aligned}
& =\left[\left\langle e\left|\sum_{n} \frac{(i t H)^{2 n}}{(2 n) !}\right| b\right\rangle+\left\langle a\left|\sum_{n} \frac{(i t H)^{2 n+1}}{(2 n+1) !}\right| b\right\rangle\right]^{\dagger} \\
& =\left[\left\langle a\left|\sum_{n} \frac{(-i t H)^{2 n}}{(2 n) !}\right| b\right\rangle-\left\langle a\left|\sum_{n} \frac{(-i t H)^{2 n+1}}{(2 n+1) !}\right| b\right\rangle\right]^{\dagger} \\
& =\left[\operatorname{Re}\left(F_{a b}\right)-i \operatorname{Im}\left(F_{a b}\right)\right]^{\dagger} \\
& \equiv \operatorname{Re}\left(F_{a b}\right)+i \operatorname{Im}\left(F_{a b}\right) \\
& \equiv F_{a b} .
\end{aligned}
$$

The preceding condition $H_{a b}=H_{b a}$ (for any two states $|a\rangle$ and $|b\rangle$ ) for a general Hamiltonian $H$, means $g_{k}=g_{k}^{*}$ in our current case. In short, one has

$$
\left\langle e,\{0\}\left|e^{-i H_{\text {eff }} t}\right| g, 1_{k}\right\rangle=\left\langle g, 1_{k}\left|e^{-i H_{\text {eff }} t}\right| e,\{0\}\right\rangle .
$$

For the second term of Eq. (B1),

$$
\sum_{k} A_{k}\left\langle e,\{0\}\left|e^{-i H_{\text {eff }} t}\right| g, 1_{k}\right\rangle,
$$

it is equal to $\alpha(t)$ for $\beta_{p}(0)=\delta_{p k}$ under Eq. (34). Thus,

$$
\begin{aligned}
\alpha & =-i \int_{0}^{t} d t^{\prime} \sum_{p} g_{p}^{\prime} \beta_{p}\left(t^{\prime}\right) e^{i\left(\Omega^{\prime}-\omega_{k}\right) t^{\prime}} \\
& =-i \int_{0}^{t} d t^{\prime} g_{k}^{\prime} e^{i\left(\Omega^{\prime}-\omega_{k}\right) t^{\prime}} \\
& =-g_{k}^{\prime} \frac{e^{i\left(\Omega^{\prime}-\omega_{k}\right) t}-1}{\Omega^{\prime}-\omega_{k}}
\end{aligned}
$$

Here we also multiply a factor $\exp \left(i \Omega^{\prime} t / 2\right)$ to remove the time-dependent factor during the transformation. Therefore,

$$
\begin{aligned}
4 \operatorname{Re} I_{\beta}(t) & =4 \operatorname{Re} \sum_{k} A_{k}\left\langle e,\{0\}\left|e^{-i H_{\mathrm{eff}} t}\right| g, 1_{k}\right\rangle e^{i \frac{\Omega^{\prime}}{2} t} \\
& =4 \operatorname{Re}\left[-i \int_{0}^{t} d t^{\prime} e^{i \Omega^{\prime} t^{\prime}} \sum_{k} A_{k} g_{k}^{\prime} e^{-i \omega_{k} t^{\prime}}\right] \\
& =4 \sum_{k} \frac{2 \Omega g_{k}^{2}}{\left(\omega_{k}+\Omega\right)^{2}} \frac{\cos \left(\Omega^{\prime}-\omega_{k}\right) t-1}{\Omega^{\prime}-\omega_{k}} \\
& =2 \pi t \int_{0}^{\infty} F\left(\omega, \Omega^{\prime}\right) G_{2}(\omega) d \omega
\end{aligned}
$$

where

$$
G_{2}(\omega)=\sum_{k} \frac{4 \Omega\left(\omega-\Omega^{\prime}\right)}{(\omega+\Omega)^{2}} g_{k}^{2} \delta\left(\omega-\omega_{k}\right) .
$$

Here we emphasize that the final result does not depend on the assumption that $g_{k}=g_{k}^{*}$. That is because it is the real parts of the second and third terms that contribute to the decay rate.

For the fourth term in Eq. (B1), we can also formally integrate $\alpha$ to have

$$
\alpha=-i \int_{0}^{t} d t^{\prime} \sum_{k} g_{k}^{\prime} \beta_{k}\left(t^{\prime}\right) e^{i\left(\Omega^{\prime}-\omega_{k}\right) t^{\prime}},
$$

and substitute it into Eq. (35) to yield

$$
\begin{aligned}
\dot{\beta}_{k} & =-\int_{0}^{t} d t^{\prime} g_{k}^{\prime} \sum_{k^{\prime}} g_{k^{\prime}} \beta_{k^{\prime}}\left(t^{\prime}\right) e^{i\left(\Omega^{\prime}-\omega_{k^{\prime}}\right) t^{\prime}} e^{-i\left(\Omega^{\prime}-\omega_{k}\right) t} \\
& =-\int_{0}^{t} d t^{\prime} e^{-i \Omega^{\prime}\left(t-t^{\prime}\right)}\left(g_{k}^{\prime}\right)^{2} e^{i \omega_{k}\left(t-t^{\prime}\right)}
\end{aligned}
$$

where we have replaced

$$
\beta_{k^{\prime}}\left(t^{\prime}\right) \simeq \beta_{k^{\prime}}(0)=\delta_{k^{\prime} k}
$$

By one more iteration, we have

$$
\beta_{k}(t)=1-\int_{0}^{t} d t_{1} \int_{0}^{t_{1}} d t_{2}\left(g_{k}^{\prime}\right)^{2} e^{i\left(\omega_{k}-\Omega^{\prime}\right)\left(t_{1}-t_{2}\right)} .
$$

Thus,

$$
\begin{aligned}
& \sum_{k} A_{k}^{2}\left\langle g, 1_{k}\left|e^{-i H_{\mathrm{eff}} t}\right| g, 1_{k}\right\rangle e^{i \frac{\Omega^{\prime}}{2} t} \\
& =\sum_{k} A_{k}^{2} \beta_{k}(t) e^{-i\left(\omega_{k}-\frac{\Omega^{\prime}}{2}\right) t} e^{i \frac{\Omega^{\prime}}{2} t} \\
& =\sum_{k} A_{k}^{2} e^{-i\left(\omega_{k}-\Omega^{\prime}\right) t}+I_{\gamma}(t) \\
& \simeq \sum_{k} A_{k}^{2} e^{-i\left(\omega_{k}-\Omega^{\prime}\right) t}
\end{aligned}
$$

where

$$
I_{\gamma}(t)=-t \int_{0}^{t} d t^{\prime}\left(1-\frac{t^{\prime}}{t}\right) \sum_{k} A_{k}^{2}\left(g_{k}^{\prime}\right)^{2} e^{-i\left(\omega_{k}-\Omega^{\prime}\right)\left(t^{\prime}-t\right)}
$$

is negligible since it is proportional to $g_{k}^{4}$.

In total,

$$
\begin{aligned}
x e^{i \frac{\Omega^{\prime}}{2} t} & =C_{1}\left(1-I_{\alpha}\right)-2 C_{2} I_{\beta}+\sum_{k} A_{k}^{2} e^{-i\left(\omega_{k}-\Omega^{\prime}\right) t} \\
& \simeq 1-I_{\alpha}-2 I_{\beta}+I_{\delta},
\end{aligned}
$$

where

$$
I_{\delta}=\sum_{k} A_{k}^{2}\left[e^{-i\left(\omega_{k}-\Omega^{\prime}\right) t}-1\right],
$$

and we have dropped higher-order terms of $\sum_{k} A_{k}^{2}$. Then, the survival probability after one measurement is

$$
\begin{aligned}
P(t) & =|x(t)|^{2} \\
& \simeq 1-2 \operatorname{Re} I_{\alpha}-4 \operatorname{Re} I_{\beta}+2 \operatorname{Re} I_{\delta} \\
& \simeq \exp \left(-2 \operatorname{Re} I_{\alpha}-4 \operatorname{Re} I_{\beta}+2 \operatorname{Re} I_{\delta}\right),
\end{aligned}
$$

with

$$
\begin{aligned}
2 \operatorname{Re} I_{\delta}(t) & =\sum_{k} \frac{-4 g_{k}^{2}}{\left(\omega_{k}+\Omega\right)^{2}} \sin ^{2} \frac{\left(\omega_{k}-\Omega^{\prime}\right) t}{2} \\
& =\sum_{k} \int_{-\infty}^{\infty} d \omega \frac{-4 g_{k}^{2} \delta\left(\omega-\omega_{k}\right)}{(\omega+\Omega)^{2}} \sin ^{2} \frac{\left(\omega-\Omega^{\prime}\right) t}{2} \\
& =-2 \pi t \int_{-\infty}^{\infty} d \omega F\left(\omega, \Omega^{\prime}\right) G_{3}(\omega)
\end{aligned}
$$

and

$$
G_{3}(\omega)=\sum_{k} \frac{\left(\omega-\Omega^{\prime}\right)^{2}}{(\omega+\Omega)^{2}} g_{k}^{2} \delta\left(\omega-\omega_{k}\right) .
$$


Straightforwardly, the survival probability after $n$ repetitive measurements is

$$
P(t)=|x(\tau)|^{2 n}=e^{-R t},
$$

where there are three contributions to the total decay rate

$$
R=R_{1}+R_{2}+R_{3},
$$

namely,

$$
\begin{aligned}
R_{1} & =2 n \operatorname{Re} I_{\alpha}(\tau) / t \\
& =2 \pi \int_{-\infty}^{\infty} d \omega F\left(\omega, \Omega^{\prime}\right) G_{1}(\omega), \\
R_{2}= & 4 n \operatorname{Re} I_{\beta}(\tau) / t \\
= & 2 \pi \int_{-\infty}^{\infty} d \omega F\left(\omega, \Omega^{\prime}\right) G_{2}(\omega), \\
R_{3}= & 2 n \operatorname{Re} I_{\delta}(\tau) / t \\
= & 2 \pi \int_{-\infty}^{\infty} d \omega F\left(\omega, \Omega^{\prime}\right) G_{3}(\omega) .
\end{aligned}
$$

To conclude, the total decay rate is further simplified as

$$
R=2 \pi \int_{-\infty}^{\infty} d \omega F\left(\omega, \Omega^{\prime}\right) G^{\prime}(\omega),
$$

where the measurement function is

$$
F\left(\omega, \Omega^{\prime}\right)=\frac{\tau}{2 \pi} \operatorname{sinc}^{2}\left(\frac{\omega-\Omega^{\prime}}{2} \tau\right),
$$

and the modified interacting spectrum is

$$
\begin{aligned}
G^{\prime}(\omega) & =G_{1}(\omega)+G_{2}(\omega)+G_{3}(\omega) \\
& =\sum_{k} f\left(\omega_{k}\right) g_{k}^{2} \delta\left(\omega-\omega_{k}\right),
\end{aligned}
$$

with the factor

$$
f\left(\omega_{k}\right)=1+\frac{\left(3 \Omega-\Omega^{\prime}+2 \omega_{k}\right)\left(\Omega-\Omega^{\prime}\right)}{\left(\omega_{k}+\Omega\right)^{2}} .
$$

[1] B. Misra and E. C. G. Sudarshan, J. Math. Phys. (NY) 18, 756 (1977).

[2] L. A. Khalfin, JETP Lett. 8, 65 (1968).

[3] M. O. Scully and M. S. Zubairy, Quantum Optics (Cambridge University Press, Cambridge, UK, 1997).

[4] E. Joos, Phys. Rev. D 29, 1626 (1984).

[5] V. Frerichs and A. Schenzle, Phys. Rev. A 44, 1962 (1991).

[6] J. J. Sakurai, Modern Quantum Mechanics (Addison-Wesley, Reading, MA, 1994).

[7] L. S. Schulman, Phys. Rev. A 57, 1509 (1998).

[8] A. G. Kofman and G. Kurizki, Nature (London) 405, 546 (2000).

[9] P. Facchi, H. Nakazato, and S. Pascazio, Phys. Rev. Lett. 86, 2699 (2001).

[10] H. Zheng, S. Y. Zhu, and M. S. Zubairy, Phys. Rev. Lett. 101, 200404 (2008).

[11] Z. H. Li, D. W. Wang, H. Zheng, S. Y. Zhu, and M. S. Zubairy, Phys. Rev. A 80, 023801 (2009).
[12] H. B. Zhu and C. P. Sun, Sci. China (A) 30, 928 (2000); Prog. Nat. Sci. 10, 698 (2000).

[13] H. Zheng, M. Avignon, and K. H. Bennemann, Phys. Rev. B 49, 9763 (1994); H. Zheng, ibid. 50, 6717 (1994).

[14] A. O. Caldeira and A. J. Leggett, Ann. Phys. 149, 374 (1983).

[15] H. Fröhlich, Phys. Rev. 79, 845 (1950); Proc. Roy. Soc. A 215 , 291 (1952); Adv. Phys. 3, 325 (1954).

[16] S. Nakajima, Adv. Phys. 4, 363 (1953).

[17] A. Blais, R. S. Huang, A. Wallraff, S. M. Girvin, and R. J. Schoelkopf, Phys. Rev. A 69, 062320 (2004).

[18] H. E. Moses, Phys. Rev. A 8, 1710 (1973).

[19] P. Facchi and S. Pascazio, Phys. Lett. A 241, 139 (1998).

[20] A. J. Leggett, S. Chakravarty, A. T. Dorsey, M. P. A. Fisher, A. Garg, and W. Zwerger, Rev. Mod. Phys. 59, 1 (1987).

[21] X. F. Cao, J. Q. You, H. Zheng, and F. Nori, e-print arXiv:1001.4831 (2010).

[22] R. Loudon and S. M. Barnett, J. Phys. B 39, S555 (2006).

[23] L. Zhou, S. Yang, Yu-xi Liu, C. P. Sun, and F. Nori, Phys. Rev. A 80, 062109 (2009). 